\title{
Influence of Femoral Implant Alignment in Uncemented Total Hip Replacement Arthroplasty: Varus Insertion and Stress Shielding
}

\author{
Hidemi Kawaji, Takuya Uematsu, Ryosuke Oba, \\ Naoya Hoshikawa, Hiroshi Watanabe and Shinro Takai \\ Department of Orthopaedic Surgery, Nippon Medical School, Tokyo, Japan
}

Background: The influence of varus insertion of femoral implants in uncemented total hip replacement arthroplasty (THR) remains unclear. Thus, in this study, we retrospectively assessed the clinical impact of uncemented THR with femoral implants that were inserted in varus on the basis of radiological findings.

Materials and Methods: The study participants included 89 patients who underwent uncemented THR for 106 joints and were followed-up for $>3$ years. From clinical records, we retrieved Japanese Orthopaedic Association (JOA) pain scores and the range of motion (ROM) of flexion and abduction both preoperatively and at the final follow-up. The presence of varus insertion of the femoral implant and stress shielding were also retrospectively reviewed from X-rays. We defined varus insertion of the femoral implant as the axis of the femoral implant that was inclined to the femoral shaft by $2^{\circ}$ or more. Stress shielding was judged in accordance with Engh's classification system.

Results: Of the 106 joints, varus insertion was observed in 40 (37.3\%) (the varus group) but not in 66 $(62.3 \%)$ (the non-varus group). The JOA pain score significantly improved in both groups; however, there were no significant differences between the groups. Although ROM improved in both groups, there were no significant differences between the groups. The appearance rate of stress shielding of $\geq$ third degree in the varus group was significantly greater than that in the non-varus group.

Conclusion: These results revealed that varus insertion of femoral implants had no influence on shortto mid-term clinical outcomes because the pain score and ROM significantly improved in both the varus and non-varus groups. However, high rates of severe stress shielding appeared with varus insertion of femoral implants, suggesting an influence on long-term clinical outcomes.

(J Nippon Med Sch 2016; 83: 223-227)

Key words: uncemented total hip replacement arthroplasty, varus insertion, stress shielding

\section{Introduction}

The long-term clinical outcomes of varus insertion of femoral implants in cemented total hip replacement arthroplasty (THR) are poor because of the prevalence of postoperative complications, such as breakage of cement in Gruen's zone 7 due to the concentration of force ${ }^{1,2}$. However, a few studies have reported that varus insertion in uncemented THR does not influence short- to mid-term clinical outcomes ${ }^{3,4}$. Therefore, we retrospectively investigated the impact of varus insertion of femoral implants, particularly on bone atrophy due to stress shielding, on the basis of clinical outcomes and radiological findings.

\section{Materials and Methods}

The study cohort included a total of 89 patients ( 9 males and 80 females) who underwent THR for 106 joints at our hospital and were followed-up for $>3$ years. The mean patient age at the time of surgery was 64.6 years, and the mean follow-up period was 6.9 years. All the surgeries were performed via the posterolateral approach with the patients in the lateral position using the VerSys

Correspondence to Hidemi Kawaji, MD, Department of Orthopaedic Surgery, Nippon Medical School, 1-1-5 Sendagi, Bunkyoku, Tokyo 113-8603, Japan

E-mail: nyao@nms.ac.jp

Journal Website (http://www.nms.ac.jp/jnms/) 
Table 1 Engh's classification: Changes in degree of severerity of resorptive bone remodeling attributable to stress shielding

\begin{tabular}{ll}
\hline $\begin{array}{l}\text { First degree } \\
\text { Second degree }\end{array}$ & $\begin{array}{l}\text { Only the most proximal medial edge of the cut femoral neck was rounded off slightly } \\
\text { Rounding off of the proximal medial femoral neck was combined with loss of medial cortical density at } \\
\text { level 1* as viewed on an anteroposterior film }\end{array}$ \\
Third degree & $\begin{array}{l}\text { More extensive resorption of cortical bone typically involved both the medial and the anterior cortical re- } \\
\text { gions at level } 1 \text { and the medial cortex at level } 2^{* *}\end{array}$ \\
Fourth degree & $\begin{array}{l}\text { Cortical resorption extended below level } 1 \text { and } 2 \text { into the diaphysis, with the changes characteristically oc- } \\
\text { curring in the medial and posterior cortices just above the level of the press fit where the cortex was most } \\
\text { widely separated from the straight stem }\end{array}$ \\
\hline
\end{tabular}

* level 1: Resorptive bone remodeling does not extend below the level of lesser trochanter.

** level 2: Resorptive bone remodeling extends below the level of lesser trochanter
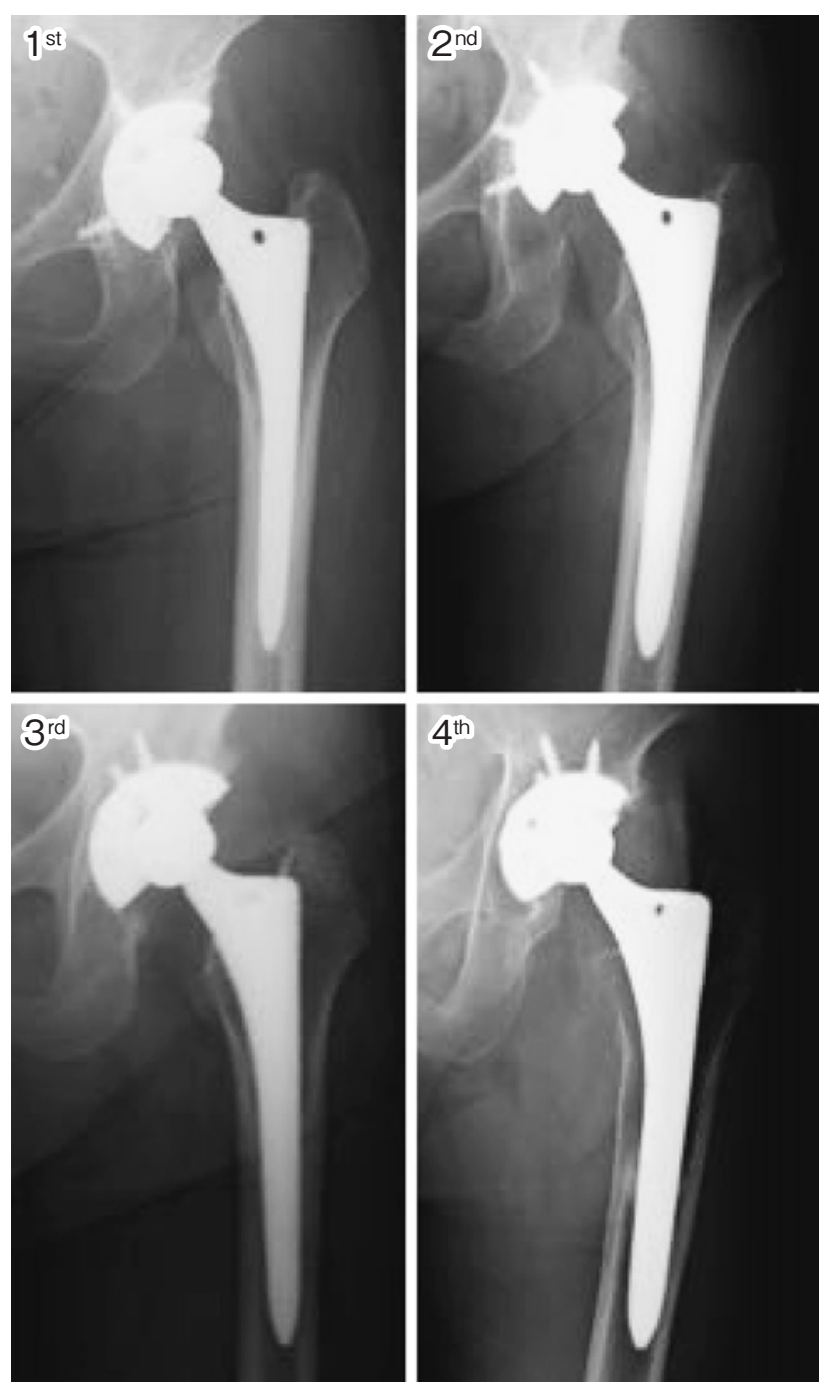

Fig. 1 Engh's classification system of stress shielding Typical X-ray photos of each degree of stress shielding are shown.

Hip System, comprising a VerSys HA/TCP Fibermetal Taper femoral implant, Trilogy Acetabular Cup Multihole, and Longevity Crosslinked Polyethylene liner (Zimmer, Warsaw, Indiana, USA).

We retrospectively reviewed clinical records to evaluate the Japanese Orthopaedic Association (JOA) pain score and the range of motion (ROM) of flexion and abduction both preoperatively and at the final follow-up. The presence of varus insertion of a femoral implant and stress shielding were also retrospectively investigated from $X$ rays. In this study, varus insertion of the femoral implant was defined as the axis of the femoral implant inclined to the femoral shaft by $2^{\circ}$ or more. Stress shielding was judged in accordance with Engh's classification system ${ }^{5}$ (Table 1, Fig. 1). The third and fourth degree in which bone resorption extended below the lesser trochanter was defined as severe stress shielding. The severity of stress shielding was categorized as the first, second, or $\geq$ third degree.

Statistical analysis was conducted using SPSS 21 (SPSS Inc, Chicago, IL, USA). The paired $t$-test was used for comparisons of clinical outcomes between the two groups, and the $\chi^{2}$ test was used to compare appearance rates. $\mathrm{P}$ value less than 0.05 was considered statistically significant.

\section{Results}

Of the 106 joints assessed in this study, 40 exhibited varus insertion of femoral implants (the varus group) and the other 66 joints did not (the non-varus group). There were no statistical differences between the patient profiles of the varus and non-varus group (4 males/36 females; mean age, 70.5 years vs. 5 males/61 females; mean age, 70.8 years, respectively). The JOA pain score and flexion/abduction ROM significantly improved in both the varus and non-varus groups at the final followup (38.5 and 38.3 points, respectively) as compared with that before the surgery (15.9 and 16.6 points, respectively); however, these differences were not significant (Fig. 2). The mean flexion angles improved from $63.8^{\circ}$ and $57.8^{\circ}$ before surgery in the varus and non-varus groups, respectively, to $90.9^{\circ}$ and $86.0^{\circ}$, respectively, at 


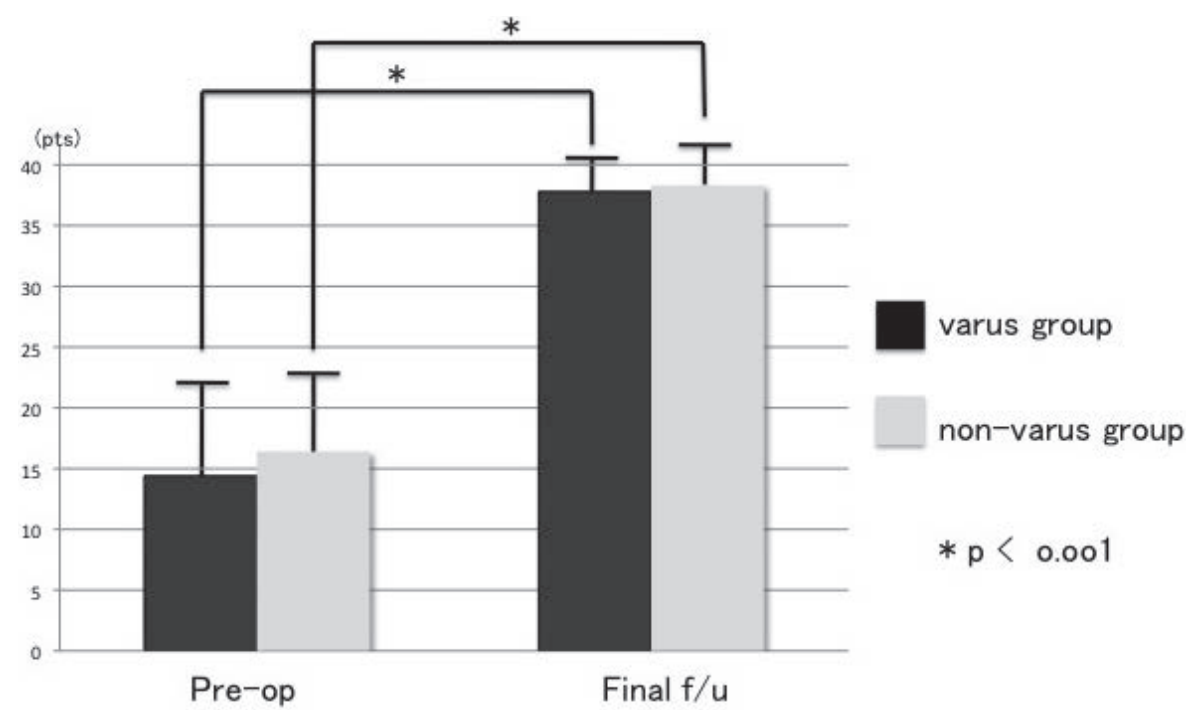

Fig. 2 JOA pain score

JOA pain score significantly improved in both groups. There were no significant differences between the two groups.

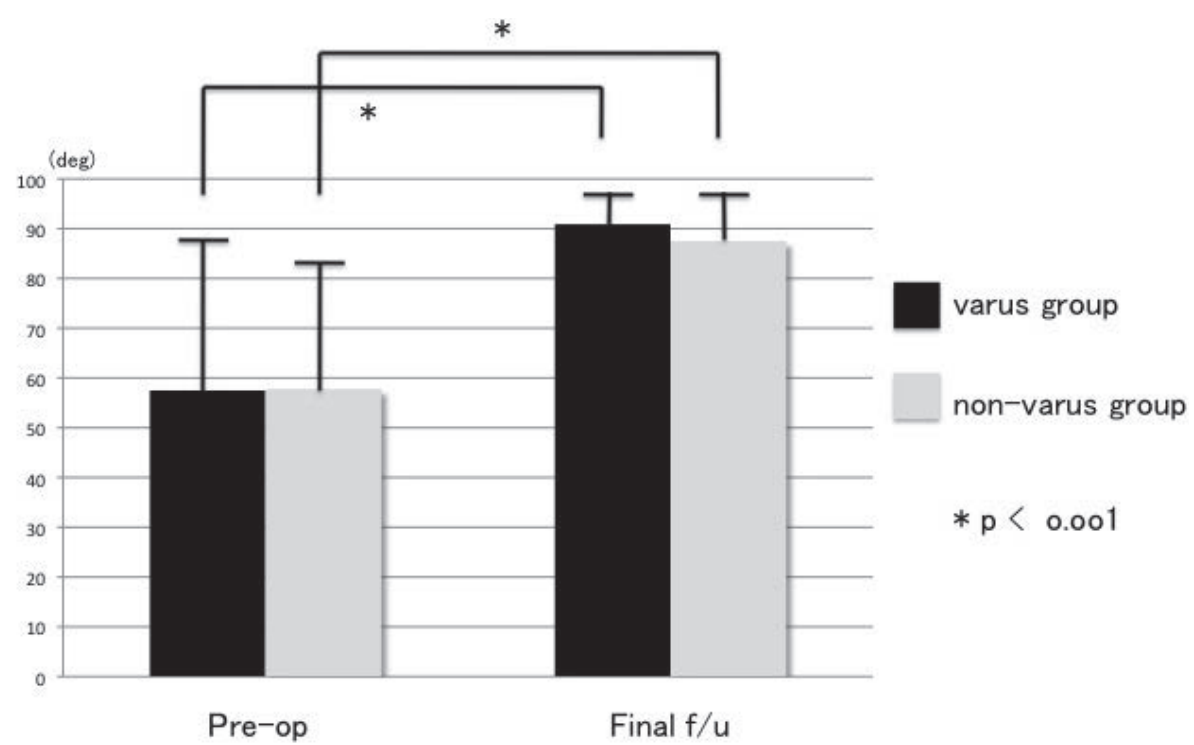

Fig. 3 ROM of flexion angle

The mean flexion angle significantly improved in both groups at the final follow-up. There were no significant differences between the two groups.

the final follow-up (Fig. 3). The mean abduction angle improved from $14.7^{\circ}$ and $10.3^{\circ}$ before the surgery in the varus and non-varus groups, respectively, to $23.8^{\circ}$ and $22.3^{\circ}$, respectively, at the final follow-up (Fig. 4). There were no significant differences in ROM measurements between the two groups. The rate of severe stress shielding of $\geq$ third degree in the varus group was significantly greater than that in the non-varus group (Table 2, Fig. 5).

\section{Discussion}

Varus insertion of a femoral implant in uncemented THR is considered to occur because the femoral implants tend to anteriorly enter into the femoral canal, resulting in flexion insertion on the lateral view. Because the tips of the femoral implants impinge on the posterior cortex of the femur in this situation, it may only be possible to insert smaller femoral implants. Accordingly, malalignment on lateral radiographs because of the small size of the femoral implants results in varus insertion on the antero- 


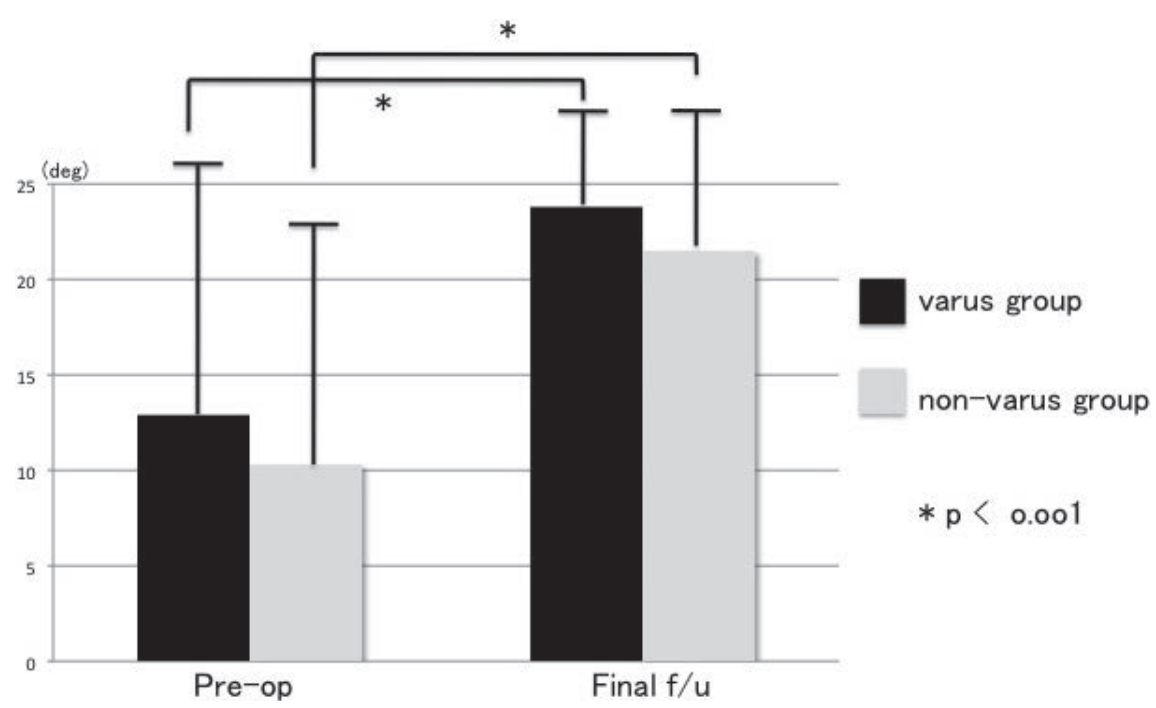

Fig. 4 ROM of abduction angle

The mean abduction angle significantly improved in both groups at the final followup. There were no significant differences between the two groups.

Table 2 Rates of stress shielding

\begin{tabular}{lcccc}
\hline & 1st degree & 2nd degree & 33rd degree & \\
\hline Varus group & 14 & 2 & 21 & joints \\
& 35.0 & 5.0 & 52.5 & $\%$ \\
\hline Non-varus group & 12 & 2 & 20 & joints \\
& 18.2 & 3.0 & 30.3 & $\%$ \\
\hline
\end{tabular}

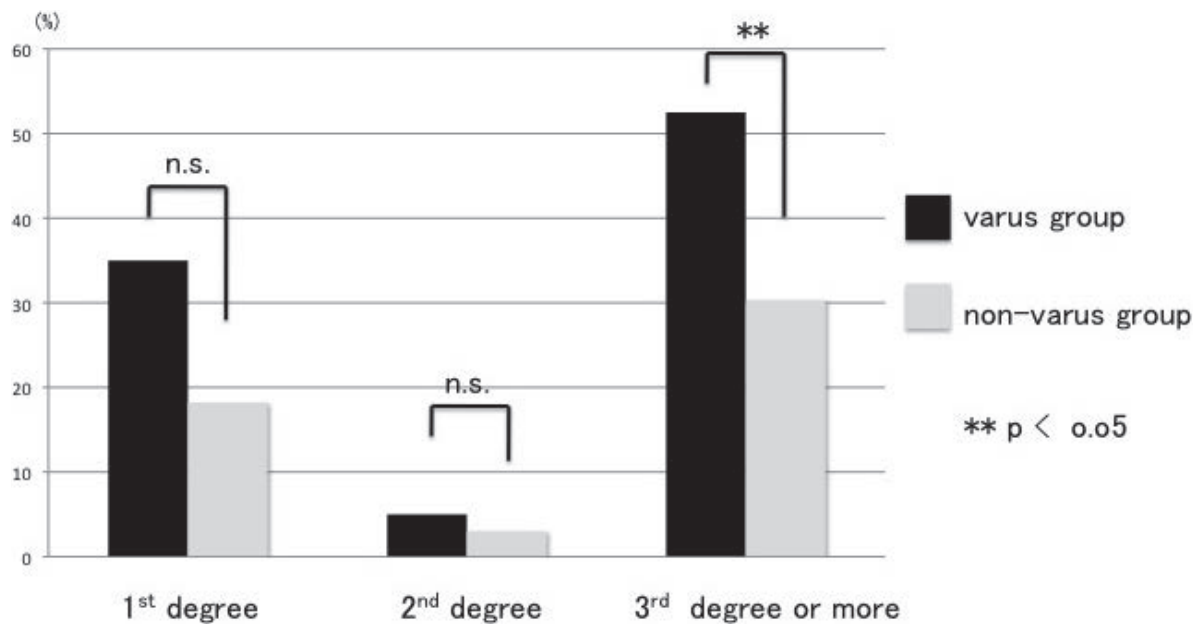

Fig. 5 Appearance rate of stress shielding

The appearance rate of stress shielding of $\geq 3$ rd degree in the varus group was significantly greater than that in the non-varus group.

posterior radiographs. To avoid malalignment on lateral radiographs, an accurate entry point to insert the femoral implants is required so that the axes of the implants align with those of the femur.

Some articles have reported that varus insertion of the femoral implants does not influence clinical outcomes; however, because these data were obtained over shortterm observational periods, long-term results remain unknown. In this study cohort, varus insertion of the femoral implants led to severe stress shielding. Thus, the fra- 
gility of the atrophied femur may have an important impact on the incidence of periprosthetic fracture or difficulty of re-operation in case of revision surgery.

Reportedly, the causes of stress shielding in uncemented THR were as follows: the size of the implant, characteristics of the implant material, property of the implant surface ${ }^{6}$, and surgical procedure. In this study, change in the transmission of the load to the femur may have occurred in cases of varus insertion of the femoral implant; thus, the surgical technique appears to be one of the most important considerations to prevent this phenomenon.

To overcome stress shielding in uncemented THR, the use of a different femoral implant material has been suggested in a study of femoral implants composed of a material that closely resembles the stiffness of natural bone ${ }^{8}$. As a more realistic approach, administration of bisphosphonate, which is used to inhibit bone resorption in osteoporosis, is reportedly effective for prevention of bone atrophy. Yamaguchi et $\mathrm{al}^{9}$ reported that etidronate disodium administration prevented stress shielding after uncemented THR. Moreover, Tapaninen et $\mathrm{al}^{10}$ reported the effectiveness of alendronate administration for the prevention of bone resoption after uncemented THR. However, Muren et $\mathrm{al}^{11}$ recently reported that administration of risedronate had no effect on periprosthetic bone atrophy. Although the effect of bisphosphonate remains controversial, if varus insertion is observed on postoperative X-rays, the administration of these drugs should be considered. Nonetheless, it is certain that avoiding varus insertion of femoral implants in the first place is the most important preventative measure.

\section{Conclusions}

The results of this study revealed that varus insertion of femoral implants had no influence on short- to mid-term clinical outcomes. However, high rates of severe stress shielding appeared with varus insertion of femoral implants, suggesting possible influences on long-term clinical outcomes.
Conflict of Interest: The authors declare no conflicts of interest.

\section{References}

1. Devitt A, O'Sulluvan T, Quinlan W: 16- to 25-year follow up study of cemented arthroplasty of the hip in patients aged 50 years or younger. J Arthroplasty 1997; 12: 479489.

2. Jaffe WL, Hawkins CA: Normalized and proportionalized cemented femoral stem survivorship at 15 years. J Arthroplasty 1999; 14: 708-713.

3. De Beer J, McKenzie S, Hubmann M, Petruccelli D, Winemaker M: Influence of cementless femoral stems inserted in varus on functional outcome in primary total hip arthroplasty. Can J Surg 2006; 49: 407-411.

4. Khality C, Lester DK: Results of tapered cementless femoral stem implanted in varus. J Arthroplasty 2002; 17: 463466.

5. Engh CA, Bobym JD, Glassman AH: Porous-coated hip replacement-The factors governing bone ingrowth, stress shielding, and clinical results. J Bone Joint Surg Br 1987; 69-B: 45-55.

6. Rubash HE, Sinha RK, Shanbhag AS, Kim SY: Pathogenesis of bone loss after total hip arthroplasty. Orthop Clin North Am 1998; 29: 173-186.

7. Nakamura N, Sugano N, Nishii T, Kakimoto A, Miki H: A comparison between robotic-assisted and manual implantation of cementless total hip arthroplasty. Clin Orthop 2010; 468: 1072-1081.

8. Caouette C, Yahia LH, Bureau MN: Reduced stress shielding with limited micromotions using a carbon fibre composite biomimetic hip stem: a finite element model. Proc Inst Mech Eng H 2011; 225: 907-919.

9. Yamaguchi K, Masuhara K, Yamasaki S, Fujii T: Efficacy of different dosing schedules of etidronate for stress shielding after cementless total hip arthroplasty. J Orthop Sci 2005; 10: 32-36.

10. Tapaninen TS, Venesmaa PK, Jurvelin JS, Miettinen HJ, Kröger HP: Alendronate reduces periprosthetic bone loss after uncemented primary total hip arthroplasty-a 5year follow-up of 16 patients. Scand J Surg 2010; 99: 3237.

11. Muren O, Akbarian E, Salemyr M, Bodén H, Eisler T, Stark A: No effect of resedronate on femoral periprosthetic bone loss following total hip arthroplasty-A 4year follow-up of 61 patients in a double blind, randomized placebo-controlled trial. Acta Orthopaedica 2015; 86: e-pub. 